\title{
Modulation-Independent Non-Data-Aided SNR Estimator for OFDM Signals in Nakagami Fading Channels
}

\author{
Mohammed Hafez \\ Department of Electrical Engineering \\ Qatar University \\ Doha, Qatar \\ Email: mohammed.hafez@qu.edu.qa
}

\author{
Tamer Khattab \\ Department of Electrical Engineering \\ Qatar University \\ Doha, Qatar \\ Email: tkhattab@ieee.org
}

\author{
Hossam Shalaby \\ Department of Electronics and \\ Communications Engineering \\ Egypt-Japan University of \\ Science and Technology (E-JUST) \\ Alexandria 21934, Egypt \\ Email: shalaby@ieee.org
}

\begin{abstract}
A signal-to-noise ratio estimator for OFDM signals in Nakagami-m fading channels is proposed. This estimator can be used with any signal constellation. It is an envelope-based non-data-aided estimator that makes use of statistical-moments of the received OFDM signals to get an estimate of the SNR in time domain. A mathematical derivation of the estimator is given and two main performance measures are evaluated, namely, the bias and the mean-squared-error. Our results reveal that this estimator works better in severe fading cases and has a good performance with low bias and low variance in the range of concern for communication systems.
\end{abstract}

Index Terms-OFDM, time-domain, SNR estimation, Nakagami fading, non-data-aided.

\section{INTRODUCTION}

Knowledge of the SNR at the receiver side is important due to its significance for several Maximum-Likelihood (ML) and Minimum-Mean-Square-Error (MMSE) techniques used in modern communication systems. The importance of knowing the instantaneous SNR increases with the use of adaptive techniques, power control, mobile-assisted handoff and feedback-assisted resources allocation. SNR estimators can be categorized into Data-Aided (DA), Decision-Directed (DD) and Non Data-Aided (NDA) estimators. DA estimators like the ones represented in [4] use the transmitted pilot symbols to get their estimates, the existence of pilot symbols causes degradation in system throughput which is the main disadvantage of this type of estimators; A DA estimator for SIMO systems is proposed in [18]. DD estimators can be considered as a special case of the DA estimators when the pilot symbols are replaced with the output of the decoder. On the other hand NDA estimators do not need any knowledge of the transmitted signal which gives it an advantage of in-service estimation. SNR estimators can be categorized in another manner; I/Q-based and envelope-based (EVB) estimators. I/Qbased estimators require coherent detection as they use inphase and quadrature components of the signal; I/Q estimators use the Expectation Maximization (EM) algorithm to get the
Maximum Likelihood (ML) estimate [16], [17]. EVB estimators can be used with an out-of-phase signal because they only make use of the signal magnitude; this can be considered as an advantage in cases where estimation is required before synchronization or equalization. Lots of EVB estimators exist for different channel models and different systems [1]-[15]. A moments-based estimator in Nakagami-m fading channel is presented in [12]; This estimator does not work with PSK signals and its formula changes with the change of the signal constellation. For OFDM signals, an estimator that makes use of some special properties of the cyclic prefix is presented in [19], another estimator for OFDM signals that depends on the frequency domain signal correlation is presented in [20]. In this paper, we use the same concept as the one of [12], but we apply it for OFDM signals. Our new estimator makes use of the time domain received signal to get an estimate for the SNR which is good for any algorithm that considers SNR as one of its inputs and works on time-domain signals. In contrast with the estimator in [12], our estimator is independent of the transmitted signal constellation, this makes it work with all types of signals including $M$-ary PSK. The rest of the paper is organized as follows. Section II describes the system model. Section III shows the derivation for the new estimator. In section IV, simulation results are presented. Finally, the conclusion is given in section $\mathrm{V}$.

\section{SySTEM MOdeL}

Consider a digital communication system over a fading channel:

$$
r_{n}=g_{n} s_{n}+w_{n}, \quad n=0, \ldots, N-1
$$

where $r_{n}$ is the received signal, $N$ is the FFT size, $g_{n}$ is the fading channel gain, $s_{n}$ is the transmitted OFDM signal and $w_{n}$ is a complex zero-mean white Gaussian noise with a variance equal to $2 \sigma_{w}^{2}$. Here $g_{n}$ is modeled as a zero-mean complex random variable and can be written as $g_{n}=\left|g_{n}\right| e^{j \phi}$ 
with $\phi$ uniformly distributed between $-\pi$ and $\pi$ and $\left|g_{n}\right|$ follows the Nakagami-m distribution:

$$
f_{\left|g_{n}\right|}\left(\left|g_{n}\right|\right)=\frac{2}{\Gamma(m)}\left(\frac{m}{\alpha_{g}^{2}}\right)^{m}\left|g_{n}\right|^{2 m-1} \exp \left(-\frac{m\left|g_{n}\right|^{2}}{\alpha_{g}^{2}}\right),
$$

where $\alpha_{g}^{2}=E\left(\left|g_{n}\right|^{2}\right), \Gamma($.$) is the Gamma function, \left|g_{n}\right|$ and $\phi$ are independent. In addition, $\left|s_{n}\right|$ can be written as:

$$
s_{n}=\frac{1}{\sqrt{N}} \sum_{k=0}^{N-1} x_{k} e^{j 2 \pi \frac{k n}{N}},
$$

where $k$ is the subcarrier index, $x_{k}$ is the baseband symbol which is taken from any arbitrary constellation.

$$
x_{k}=I_{k}+j Q_{k}
$$

where $I_{k}$ and $Q_{k}$ are in-phase and quadrature components respectively. According to the central limit theorem and with a large $N$ and assuming that $x_{k}$ has zero-mean and the constellation power is normalized, it can be considered that $s_{n}$ is a complex Gaussian random variable with zero-mean and normalized power. This makes $\left|s_{n}\right|$ follows the Rayleigh distribution.

$$
f_{\left|s_{n}\right|}\left(\left|s_{n}\right|\right)=\frac{\left|s_{n}\right|}{\sigma_{s}^{2}} e^{-\frac{\left|s_{n}\right|^{2}}{2 \sigma_{s}^{2}}}
$$

where $\sigma_{s}^{2}=\operatorname{Var}\left(\left|s_{n}\right|\right)=\frac{1}{2}$ following the assumptions stated above. So, the SNR can be expressed as:

$$
\rho=\frac{\alpha_{g}^{2}}{2 \sigma_{w}^{2}}
$$

\section{Proposed Estimator}

The idea is to get an estimate of the SNR using the ratio between the different moments of the received time-domain signal. The probability density function of $r_{n}$, conditioned on both $g_{n}$ and $s_{n}$, is given by [21]:

$$
\begin{aligned}
p\left(\left|r_{n}\right| \mid g_{n}, s_{n}\right)= & \frac{\left|r_{n}\right|}{\sigma_{w}^{2}} \\
& \exp \left(-\frac{\left|g_{n}\right|^{2}\left|s_{n}\right|^{2}+\left|r_{n}\right|^{2}}{2 \sigma_{w}^{2}}\right) \\
& \times I_{0}\left(\frac{\left|r_{n}\right|\left|g_{n}\right|\left|s_{n}\right|}{\sigma_{w}^{2}}\right),
\end{aligned}
$$

where $I_{0}($.$) is the modified bessel function of the first kind$ and the order zero. This leads to:

$$
\begin{aligned}
E\left[\left|r_{n}\right|^{l}\right]= & \int_{0}^{\infty} 2^{\frac{l}{2}} \Gamma\left(\frac{l}{2}+1\right) \sigma_{w}^{l} e^{-\left|s_{n}\right|^{2}}\left(\frac{m}{m+\rho\left|s_{n}\right|^{2}}\right)^{m} \\
& \times{ }_{2} F_{1}\left(\frac{l}{2}+1, m, 1 ; \frac{\rho\left|s_{n}\right|^{2}}{m+\rho\left|s_{n}\right|^{2}}\right) d\left|s_{n}\right|^{2}
\end{aligned}
$$

where ${ }_{2} F_{1}(a, b, c ; x)$ is the confluent hypergeometric function.

Here we will use the relation between fourth and second moments of the signal to get the estimate of the SNR. Defining $z$ as the estimation parameter, we get:

$$
z=\frac{M_{4}}{M_{2}^{2}}=f(\rho)
$$

$$
\begin{gathered}
M_{l}=E\left[\left|r_{n}\right|^{l}\right], \\
\rho=f^{-1}(z)
\end{gathered}
$$

as $M_{l}$ is not available in practice, we can use the following estimate using the received signal samples:

$$
\begin{gathered}
\hat{M}_{l}=\frac{1}{N} \sum_{n=0}^{N}\left|r_{n}\right|^{l}, \\
\hat{z}=\frac{\hat{M}_{4}}{\hat{M}_{2}^{2}},
\end{gathered}
$$

And so

$$
\hat{\rho}=f^{-1}(\hat{z})
$$

The inverse function can be implemented by using lookup tables, polynomial approximation or direct formula. The independence of this estimator of the signal constellation gives the estimator the advantage of the use in adaptive systems as only one inverse function is needed for all different constellations used by the system.

\section{A. Special case: $m=1$ (i.e., Rayleigh fading)}

$E\left[\left|r_{n}\right|^{l}\right]=\int_{0}^{\infty} 2^{\frac{l}{2}} \Gamma\left(\frac{l}{2}+1\right) \sigma_{w}^{l} e^{-\left|s_{n}\right|^{2}}\left(1+\rho\left|s_{n}\right|^{2}\right)^{\frac{l}{2}} d\left|s_{n}\right|^{2}$

$$
\begin{aligned}
M_{2} & =2 \sigma_{w}^{2} \int_{0}^{\infty} e^{-\left|s_{n}\right|^{2}}\left(1+\rho\left|s_{n}\right|^{2}\right) d\left|s_{n}\right|^{2} \\
& =2 \sigma_{w}^{2}(1+\rho) \\
M_{4}=8 \sigma_{w}^{4} \int_{0}^{\infty} e^{-\left|s_{n}\right|^{2}}\left(1+\rho\left|s_{n}\right|^{2}\right)^{2} d\left|s_{n}\right|^{2} & \\
= & 8 \sigma_{w}^{4} \int_{0}^{\infty} e^{-\left|s_{n}\right|^{2}}\left(1+2 \rho\left|s_{n}\right|^{2}+\left(\rho\left|s_{n}\right|^{2}\right)^{2}\right) d\left|s_{n}\right|^{2} \\
= & 8 \sigma_{w}^{4}\left(1+2 \rho+2 \rho^{2}\right),
\end{aligned}
$$

Then

$$
\begin{gathered}
z=2 \frac{\left(1+2 \rho+2 \rho^{2}\right)}{\left(1+2 \rho+\rho^{2}\right)}, \\
\rho=\frac{z-2}{4-z}+\frac{\sqrt{2} \sqrt{z-2}}{4-z}, \quad 2<z<4
\end{gathered}
$$




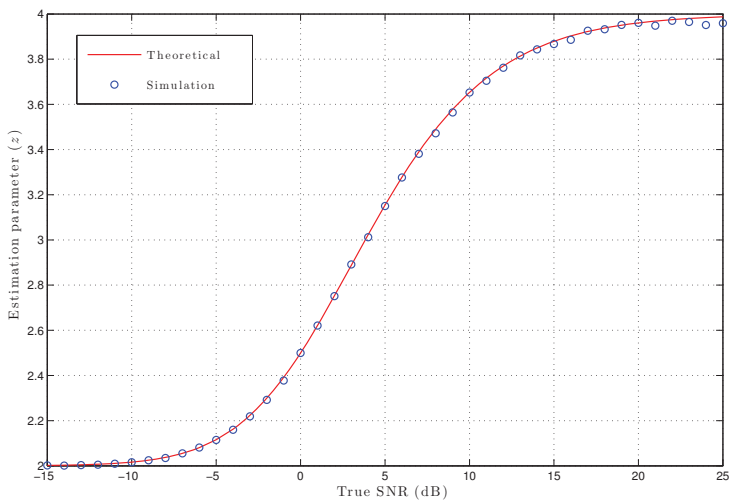

Fig. 1. Relation between the estimation parameter $z$ and SNR.

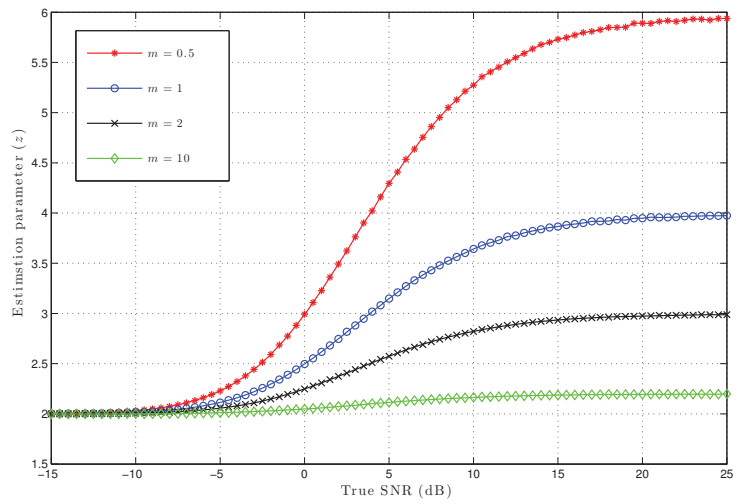

Fig. 2. Relation between the estimation parameter $z$ and SNR for different values of Nakagami-m parameter.

\section{RESUlts}

The simulation is done using FFT size $N=2^{14}, 16-\mathrm{QAM}$ signal and $m=1$, unless something else is stated. In Fig. 1, we draw the relation between the estimation parameter $z$ and the SNR, one time by simulation using (13) and the other time depending on the theoretical solution from (18), both cases are almost identical.

Figure 2 shows the same relation but for different values of the Nakagami-m parameter, it can be seen that the range of change decreases with the increase of $m$, this means that the estimator would have a better performance in severe fading cases, i.e., the estimator would not work in the AWGN channel (the case where $m \rightarrow \infty$ ). Also from the figure we can recognize that the relation saturates for very high and very low SNR values. That is, the change occurs in the range from -5 $\mathrm{dB}$ to $15 \mathrm{~dB}$ which is the range of concern for communication systems.

In Fig. 3, we used two different baseband constellations $Q$ PSK and 64-QAM to show that the change of the signal constellation has no effect on the relation. Also the figure presents the relation in the frequency-domain, which is constant over the whole range of the SNRs. This means that we cannot use
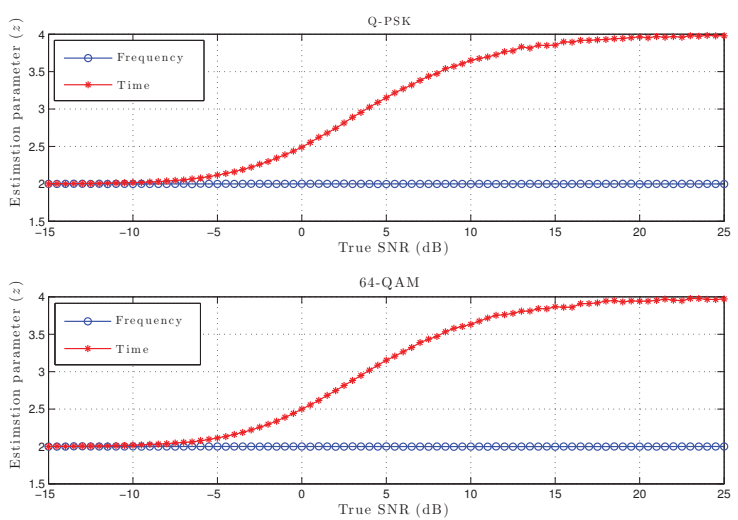

Fig. 3. Relation between the estimation parameter $z$ and the SNR for $Q$-PSK and 64-QAM signals in both time- and frequency-domains.

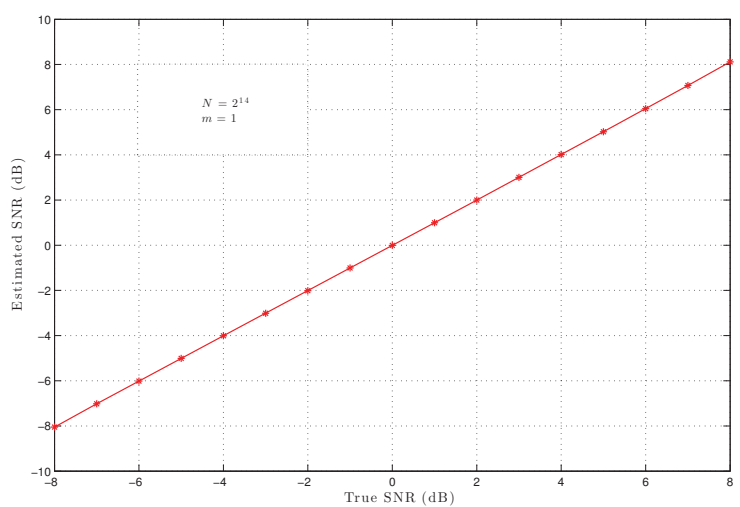

Fig. 4. Average estimated SNR versus true SNR.

the same estimation concept for frequency-domain signals.

Figures 4, 5, and 6 evaluate the performance of our estimator. The estimator has a good performance in the range form $-8 \mathrm{~dB}$ to $8 \mathrm{~dB}$, it is unbiased and have low error probability. After this range the performance starts to degrade.

In Fig. 4, we draw the estimated SNR compared to the true $\mathrm{SNR}$, the estimator produces an exact estimation in the range of the good performance.

Figure 5 represents the bias of the estimator, which is the expected value of the estimator error, the estimator can be considered unbiased in the low SNR range and the bias increases with high SNR values.

In Fig. 6, we plot the Normalized-Root-Mean-SquaredError (NRMSE) for different signal constellations, which is normalized by the investigated SNR range. In addition to the low bias of the estimator in the low SNR range, the estimator has low MSE, which means that it has a low variance, as well. This makes the estimation results in Fig. 4 reasonable; moreover, the NRMSE takes random values in the high SNR range. This randomness can be clarified by refereing to Fig. 1 , it can be recognized that the non-linear part of the curve spans a wide range which means that any small variation 


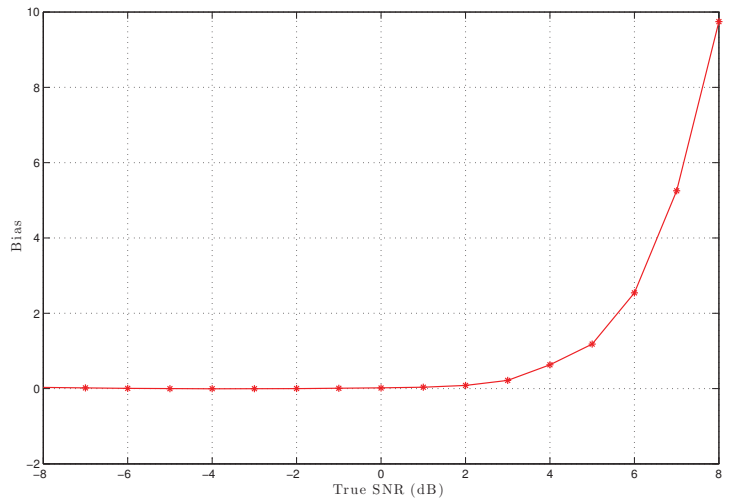

Fig. 5. Average bias of the estimator versus true SNR.

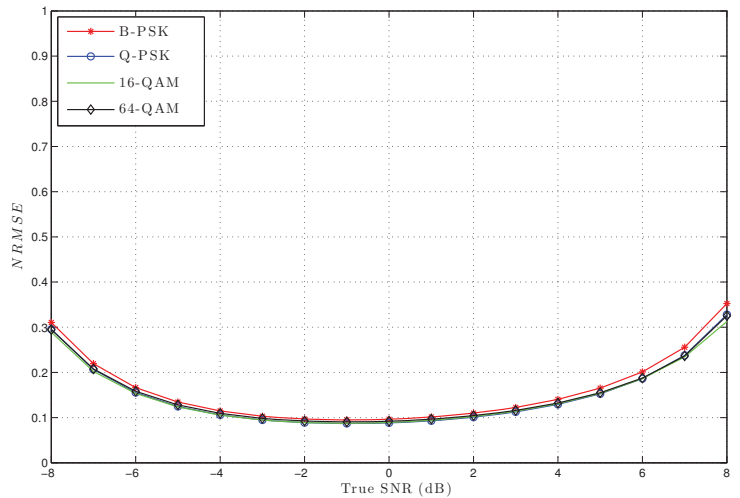

Fig. 6. Average NRMSE of the estimator versus true SNR.

in the statistical estimates would cause a large error in the estimated SNR. Also the simulated curve has more errors in the high SNR range. This figure is another mean of proving the independence of the estimator of the modulation scheme.

\section{CONCLUSION}

A new estimator, that can get an estimate of the SNR for OFDM signals in time-domain with no need for knowledge of the baseband constellation, has been proposed. A mathematical derivation of the estimator has been given and two main performance measures have been evaluated, namely, the bias and the mean-squared-error of the estimator. Our results reveal that this estimator works better in the more severe fading cases. Indeed, it has a good performance with low bias and low variance in the range of concern for communication systems.

\section{ACKNOWLEDGEMENT}

The research work in this paper has been funded by QNRF grant number NPRP 09-231-2-095.

\section{REFERENCES}

[1] T. A. Summers and S. G. Wilson, "SNR mismatch and online estimation in Turbo decoding," IEEE Transactions on Communications, vol. 46, no. 4, pp. 421-423, April 1998.
[2] H. Xu, Z. Li, and H. Zheng, "A non-data-aided SNR estimation algorithm for QAM signals," International Conference on Communications, Circuits and Systems, vol.2, pp. 999-1003, June 2004.

[3] O. H. Tekbas, "Blind SNR estimation for limited time series," Chaos, Solitons and Fractals, vol. 33, no. 5, pp. 1497-1504, August 2007.

[4] D. R. Pauluzzi and N. C. Beaulieu, "A comparison of SNR estimation techniques for the AWGN channel," IEEE Transactions on Communications, vol. 48, no. 10, pp. 1681-1691, October 2000.

[5] R. Matzner, "An SNR estimation algorithm for complex baseband signals using higher order statistics," Facta Universitatis (Nis), vol. 6, no.1, pp. 4152, 1993.

[6] A. Wiesel, J. Goldberg, and H. Messer, "Non-data-aided signal-to-noiseratio estimation," IEEE International Conference on Communications, vol. 1, pp. 197-201, 2002.

[7] A. Wiesel, J. Goldberg, and H. Messer, "SNR estimation in time-varying fading channels," IEEE Transactions on Communications, vol. 54, no. 5, pp. 841-848, May 2006.

[8] R. Lpez-Valcarce, C. Mosquera, and W. Gappmair, "Iterative envelopebased SNR estimation for non-constant modulus constellations," IEEE 8th Workshop on Signal Processing Advances in Wireless Communications, pp. 1-5, June 2007.

[9] P. Gao and C. Tepedelenlioglu, "SNR estimation for non-constant modulus constellations," IEEE Transactions on Signal Processing, vol. 53, no.3, pp. 865-870, March 2005.

[10] M. lvarez-Daz, R. Lpez-Valcarce, and C. Mosquera, "SNR estimation for multilevel constellations using higher-order moments," IEEE Transactions on Signal Processing, vol. 58, no.3, pp. 1515-1526, March 2010.

[11] A. Ramesh, A. Chockalingam, and L. B. Milstein, "SNR estimation in generalized fading channels and its application to Turbo decoding," IEEE International Conference on Communications, vol. 4, pp. 10941098, 2001.

[12] S. A. Dianat, "SNR estimation in Nakagami fading channels with arbitrary constellation," IEEE International Conference on Acoustics, Speech and Signal Processing, vol. 2, pp. 325-328, April 2007.

[13] A. Stphenne, F. Bellili, and S. Affes, "Moment-based SNR estimation over linearly-modulated wireless SIMO channels," IEEE Transactions on Wireless Communications, vol. 9, no. 2, pp. 714-722, February 2010.

[14] A. Ramesh, A. Chockalingam, and L. B. Milstein, "SNR estimation in Nakagami-m fading with diversity combining and its application to Turbo decoding," IEEE Transactions on Communications, vol. 50, no. 11, pp. 1719-1724, November 2002.

[15] R. Lpez-Valcarce and C. Mosquera, "Sixth-order statistics-based nondata-aided SNR estimation," IEEE Communications Letters, vol. 11, no. 4, pp. 351-353, April 2007.

[16] A. Das, "NDA SNR estimation: CRLBs and EM based estimators," IEEE Region 10 Conference, pp. 1-6, November 2008.

[17] J. Descure, F. Bellili, and S. Affes, "ML estimator based on the EM algorithm for subcarrier SNR estimation in multicarrier transmissions," IEEE AFRICON, pp. 1-5, September 2009.

[18] F. Bellili, A. Stephenne, and S. Affes, "SNR estimation of QAMmodulated transmissions over time-varying SIMO channels," IEEE International Symposium on Wireless Communication Systems, pp. 199203, October 2008.

[19] F. Socheleau, A. Assa-El-Bey, and S. Houcke, "Non data-aided SNR estimation of OFDM signals," IEEE Communications Letters, vol. 12, no. 11 , pp. 813-815, November 2008.

[20] S. A. Kim, D. G. An, H. Ryu, and J. Kim, "Efficient SNR estimation in OFDM system," IEEE Radio and Wireless Symposium, pp. 182-185, January 2011.

[21] J. G. Proakis, "Digital Communications", 4ed., New York: McGrawHill, 2001. 